\title{
General approach of the photothermoelectric technique for thermal characterization of solid thermoelectric materials
}

Karim Touati, ${ }^{1,2}$ Michael Depriester, ${ }^{1,2}$ Emmanuel Guilmeau, ${ }^{3}$ Andrés Sotelo, ${ }^{4}$ Maria A. Madre, ${ }^{4}$ Franck Gascoin, ${ }^{3}$ and Abdelhak Hadj Sahraoui ${ }^{1,2}$

1) UDSMM (EA 4476), MREI-1, Université du Littoral Côte d'Opale, 59140 Dunkerque, France

2) Univ Lille Nord de France, 59044 Lille, FRANCE

3) CRISMAT, UMR 6508 CNRS/ENSICAEN, 6 Bd du Maréchal Juin, F-14050 Caen, France

4) Instituto de Ciencia de Materiales de Aragón (CSIC-Universidad de Zaragoza), $M$ de Luna, 3, 50018 Zaragoza, Spain

This work focuses on the photothermoelectric (PTE) technique allowing the thermal characterization of solid-state thermoelectric (TE) materials. Previously, this technique was restricted to TE materials having low electrical conductivities. Here, the PTE technique is extended and generalized to all solid-state TE materials with low or high electrical conductivities. This is achieved by taking into account the Gaussian shape of the thermal excitation source. The formalism of this new methodology is developed and the procedure for extracting thermal parameters is proposed. For illustration, two different TE materials are studied: with relatively high electrical conductivity $\left(\mathrm{Bi}_{2} \mathrm{Te}_{2.4} \mathrm{Se}_{0.6}\right)$ and relatively low electrical conductivity $\left(\mathrm{Bi}_{2} \mathrm{Ca}_{2} \mathrm{Co}_{1.7} \mathrm{O}_{x}\right)$. The thermal properties of these two materials (thermal diffusivity, effusivity and conductivity) are found and compared to those obtained by the photothermal radiometry which is a well established technique. The good concordance between the results obtained by these two techniques demonstrates the relevance of the generalized PTE technique. One of the main advantages of this technique is its non use of an external sensor.

Keywords: Seebeck effect, Thermoelectric materials, Thermal conductivity, Photothermoelectricity 


\section{INTRODUCTION}

Because of the energy challenge facing our planet, a lot of research on renewable and environment friendly resources is taking place around the world. Given that more than two-thirds of the energy produced by man in the various industrial systems (Nuclear plant, Automobile, etc.) is lost in form of heat, more and more research organizations are interested in the recovering and valorizing of this fatal energy. Among the most explored avenues, thermoelectric (TE) generators are attracting a particular interest. This technology presents many advantages: the non-emission of pollutant gas, the non-existence of any audible noise as a consequence of the absence of moving part, etc. Thermoelectric generators are mostly solidstate materials allowing direct conversion of thermal energy into electricity due to Seebeck effect.

The majority of researches carried out these last years on thermoelectric materials focus on improving their performances. The conversion efficiency of heat into electricity of TE materials is a function of their figure of merit given by ${ }^{1} Z T=\left(S^{2} \sigma_{e} / \kappa\right) T$. This dimensionless parameter is dependent on the Seebeck coefficient $(S)$, the electrical conductivity $\left(\sigma_{e}\right)$, the thermal conductivity $(\kappa)$ and the average absolute temperature of the studied material. The improvement can be achieved by increasing their power factor $\left(S^{2} \sigma_{e}\right)$ or by reducing their thermal conductivity $(\kappa)$. Since the electronic contribution to the thermal conductivity $\left(\kappa_{e}\right)$ is directly linked to the electrical conductivity by the Wiedemann-Franz law, the reduction of this electronic contribution will conduct to a similar evolution of the electrical conductivity. As the lattice contribution of the thermal conductivity $\left(\kappa_{l}\right)$ is not correlated with $\sigma_{e}$, more and more researches deal with the reduction of this contribution.

So, an accurate determination of the thermal and electrical properties is necessary for the improvement of TE materials performance. Although the measurement of Seebeck coefficient and electrical conductivity are often reported, thermal conductivity of these materials is scarcely measured directly. It is commonly calculated by the combination of thermal diffusivity $(\alpha)$, heat capacity $\left(C_{p}\right)$ and density $(\rho)$ of these materials, which may not be as accurate as a direct measurement. For this purpose, the photothermoelectric (PTE) technique was developed $^{2-4}$. This original technique allows a direct and accurate thermal characterization of TE materials without using an external sensor. It is based on a self-generated voltage 
due to the Seebeck effect. This voltage is provided by the sample when one of its two faces is thermally excited using a modulated laser at a given frequency. Access to thermal parameters is possible through analysis of the signal (amplitude and phase) evolution as function of the exciting frequency. Thermal diffusivity and effusivity $(e)$ can be simultaneously determined. The combination of these two parameters allows the calculation of the TE material thermal conductivity using the relation $\kappa=e \sqrt{\alpha}$. This technique was used to study thermally many solid TE materials having low electrical conductivities. Among these we can cite the polyaniline/carbon nanotube composites and titanium trisulfide $\left(\mathrm{TiS}_{3}\right)$.

Until now, The $\mathrm{PTE}_{1 D}$ model presented in reference ${ }^{2}$ was restricted to TE materials presenting low electrical conductivities. This model showed its limits when we were interested in the thermal characterization of TE materials having relatively high electrical conduc-tivities such as the bismuth selenido telluride $\left(\mathrm{Bi}_{2} \mathrm{Te}_{2.4} \mathrm{Se}_{0.6}\right)$. Indeed, this model doesn't fit the experimental data delivered by such type of materials. To remedy this issue, a new photothermoelectric model allowing the thermal characterization of all TE materials (having low or high electrical conductivity) is developed. The followed procedure and the new developed model are presented in the present work.

\section{THEORETICAL APPROACH}

\section{A. Simulation of the electrical potential distribution in a TE material subjected to a Gaussian modulated laser beam}

The PTE technique is based on the voltage appearing between the two faces of a TE material kept at different temperatures $(\Delta \bar{T})$. This voltage is given by:

$$
\Delta \bar{V}=-S \Delta \bar{T}
$$

To determine the voltage distribution, the temperature expression at each face of the TE material is needed. This expression can be obtained from the resolution of the heat equation. Since the thermal excitation source is not homogeneous (laser beam having a Gaussian shape), highly conductive thin layers (gold) are deposited on the two sides of the studied TE sample. This allows the homogenization of charges at the sample surfaces. 
Then a one dimensional (1D) heat propagation can be considered. This is achieved in the case of a low electrical conductive TE materials but not in the case of high electrical conductive TE materials. To visualize the effect of these two conductive layers, the electrical potential distribution in a low and high electrical conductive TE material will be simulated.

From the Onsager relations ${ }^{5}$, heat and electric charge continuity equations ${ }^{6}$, we established two coupled equations (eq.(2) and eq.(3)) describing all the thermal and electrical phenomena encountered in a TE material subjected to a modulated thermal excitation.

$$
\begin{gathered}
\rho c_{p} j \omega \bar{T}-\sigma_{e} S T \nabla^{2} \bar{V}-\left(\sigma_{e} S^{2} T+\kappa_{J}\right) \nabla^{2} \bar{T}=\overline{\dot{q}} \\
\sigma_{e} \nabla^{2} \bar{V}+\sigma_{e} S \nabla^{2} \bar{T}+j \omega \epsilon \nabla^{2} \bar{V}=0
\end{gathered}
$$

with $j^{2}=-1, \omega=2 \pi f, f$ is the excitation frequency. $\bar{T}$ and $\bar{V}$ are respectively the modulated part of temperature and electric potential across the TE material. $\epsilon$ is the material dielectric permittivity. $\dot{q}$ is the heat generation rate per unit volume $\left[\mathrm{W} \cdot \mathrm{m}^{-3}\right]$.

The electrical potential distribution in a TE material subjected to a Gaussian laser beam can be simulated by solving these two coupled equations. For this purpose, the Matlab solver called PDE (Partial Differential Equations) based on the finite element method is used. Simulations are performed for low and high electrical conductive TE material by considering the system represented in Figure 1. In both simulations, the thicknesses $\left(L_{1}\right)$ of TE samples and the substrate (water) are taken equal to $450 \mu \mathrm{m}$ and to $\sim 3 \mathrm{~mm}$, respectively. The thickness of the substrate is taken sufficiently large to prevent that the thermal wave reaches its rear side, as a consequence the Dirichlet conditions are imposed at this location. For the lateral sides, Neumann conditions are imposed. To observe the effect of the coating on the electrical potential distribution, in both cases a thin gold layer having a thickness of $10 \mu \mathrm{m}$ is used. The physical parameters of the different layers involved in theses simulations are given in table I. In order to be closer to our experimental conditions, the laser beam radius $\left(R_{l}\right)$ is taken equal to $6 \mathrm{~mm}$ and the excitation frequency $(f)$ is chosen in the range where the sample is thermally thin $(1 \mathrm{~Hz})$. In these simulations we assumed that $\dot{q}$ is equal to zero. 


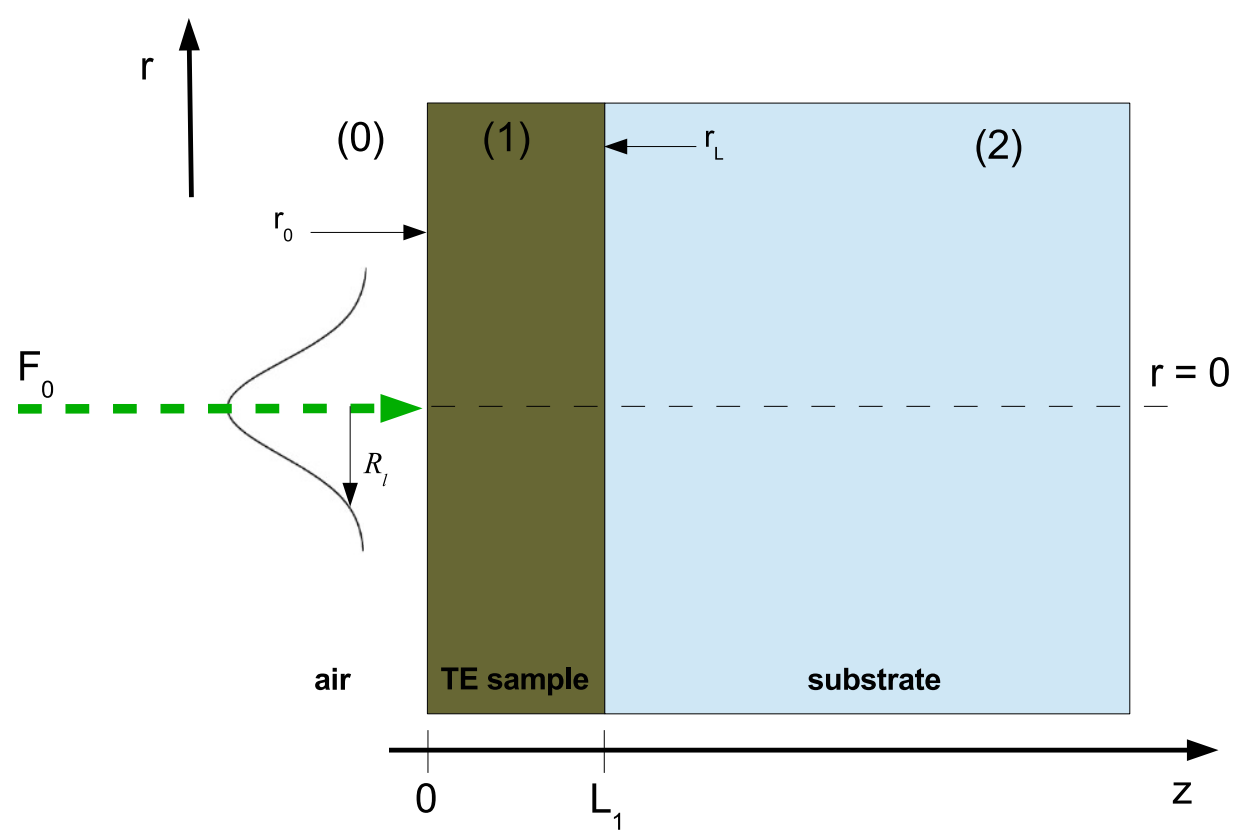

Figure 1. Geometry of the studied system used for the simulation and the modelization of the photothermoelectric signal.

Figure 2 exhibits the electric potential distribution in a TE material $\left(\mathrm{Bi}_{2} \mathrm{Ca}_{2} \mathrm{Co}_{1.7} \mathrm{O}_{x}\right)$, presenting a relatively low electrical conductivity, and in the substrate on which this sample is placed. As it was expected, this figure shows that the electrical potential distribution in the TE material follows the shape of the excitation thermal source (Gaussian shape). In this case, the assumption of a 1D heat propagation is not satisfied due to this inhomogeneous distribution. Then, thin gold layers are deposited at the sample faces. As a consequence, a complete electrical potential homogenization can be observed at the two faces, as it is shown on Figure 3. 


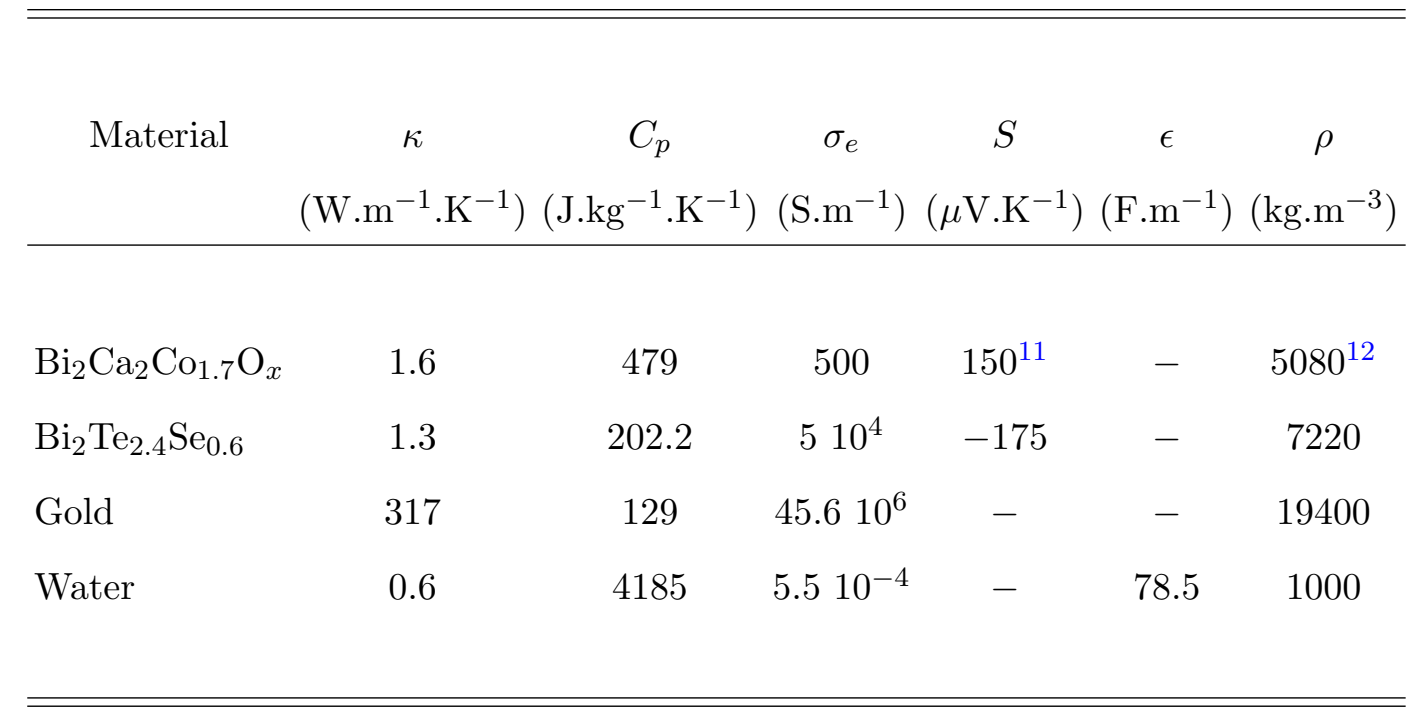

Table I. Physical parameters of the two thermoelectric materials simulated in this section $\left(\mathrm{Bi}_{2} \mathrm{Ca}_{2} \mathrm{Co}_{1.7} \mathrm{O}_{x}, \mathrm{Bi}_{2} \mathrm{Te}_{2.4} \mathrm{Se}_{0.6}\right)$ and those of gold used as electrodes and water used as substrate at ambient temperature $\left(20^{\circ} \mathrm{C}\right)$.

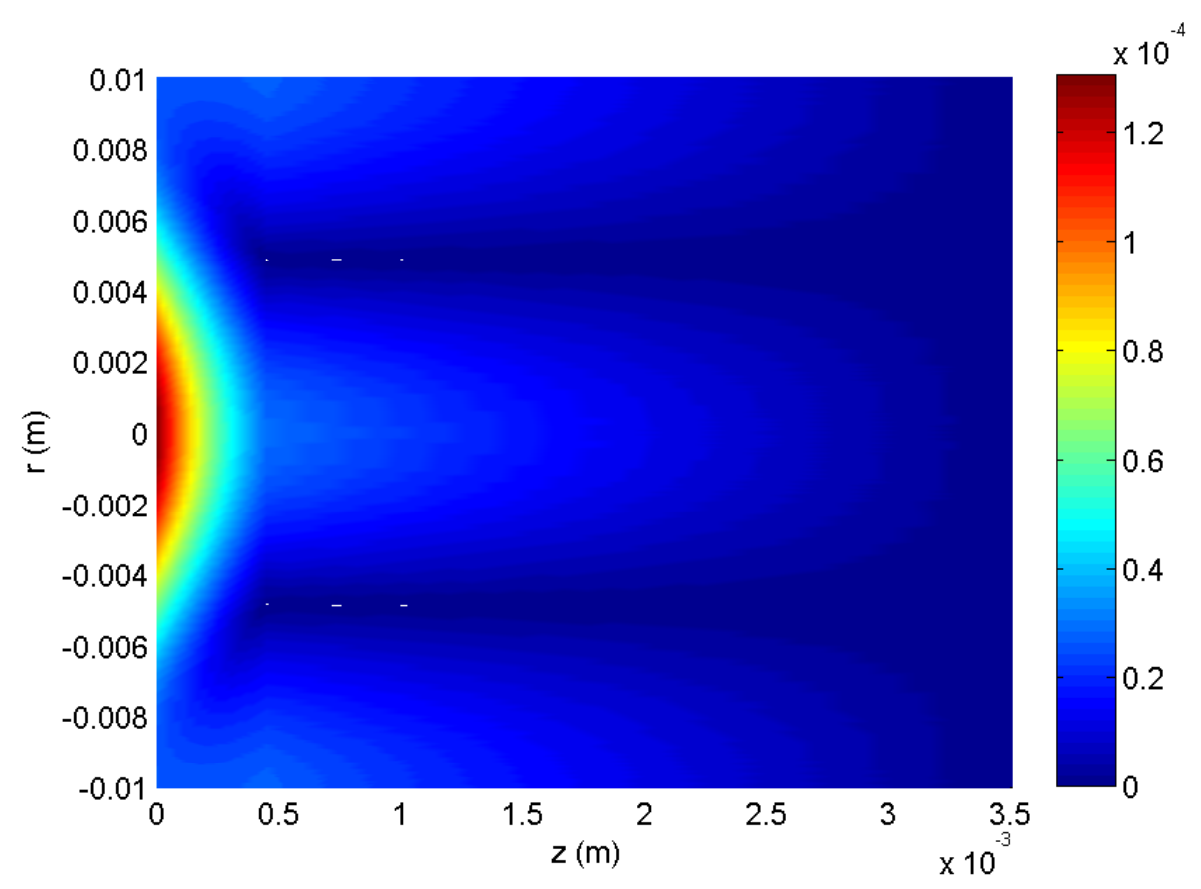

Figure 2. Simulation of the electrical potential amplitude (V) distribution in a thermoelectric material presenting low electrical conductivity $\left(\mathrm{Bi}_{2} \mathrm{Ca}_{2} \mathrm{Co}_{1.7} \mathrm{O}_{x}\right)$ subjected to a modulated Gaussian thermal excitation. 


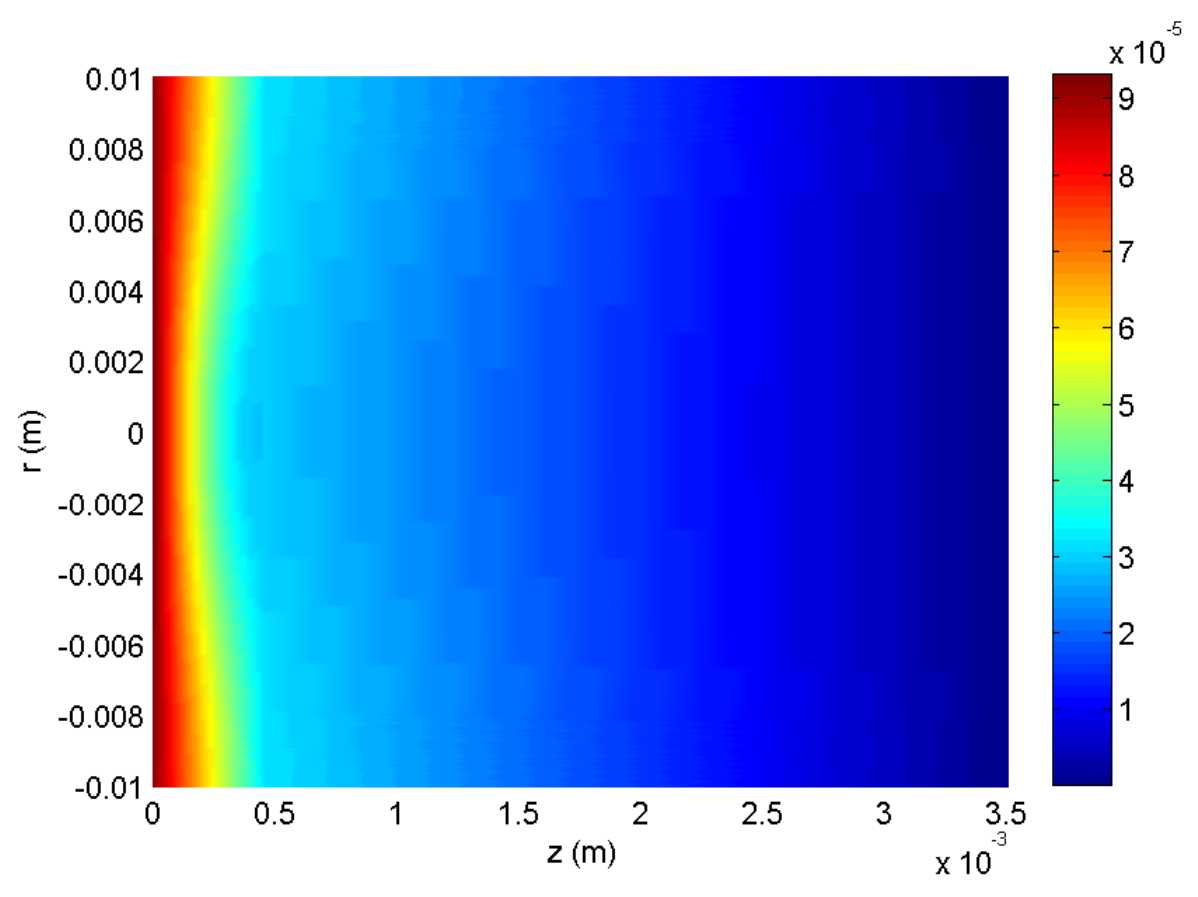

Figure 3. Simulation of the influence of the deposition of two gold layers on the electrical potential amplitude (V) distribution in a $\mathrm{Bi}_{2} \mathrm{Ca}_{2} \mathrm{Co}_{1.7} \mathrm{O}_{x}$ sample subjected to a modulated Gaussian thermal excitation.

Now, the coating effect on the electrical potential distribution in the case of a high conductive material $\left(\mathrm{Bi}_{2} \mathrm{Te}_{2.4} \mathrm{Se}_{0.6}\right)$ will be highlighted.

Figure 4 shows that the gold layers deposited on a TE material having a $\sigma_{e}=510^{4}{\mathrm{~S} . \mathrm{m}^{-1}}^{-1}$ allow a better electrical potential distribution but not a complete charges homogenization at the sample faces. Consequently, an influence of the electrical contacts position allowing the photothermoelectric signal collection appears. The access to thermal properties of such TE materials by the PTE technique can be achieved by following two ways. The first consists on depositing a thicker gold layer allowing a complete potential homogenization on the sample faces. In this case, the $\mathrm{PTE}_{1 D}$ model can be used. The second way to follow consists on developing a new PTE model taking into account the positions of the electrical contacts on the sample faces.

Because the first solution is economically unsustainable (a huge gold quantity is needed to reach a complete potential homogenization), the second solution will be retained. We propose in the following section a new theoretical model allowing the thermal characteriza- 


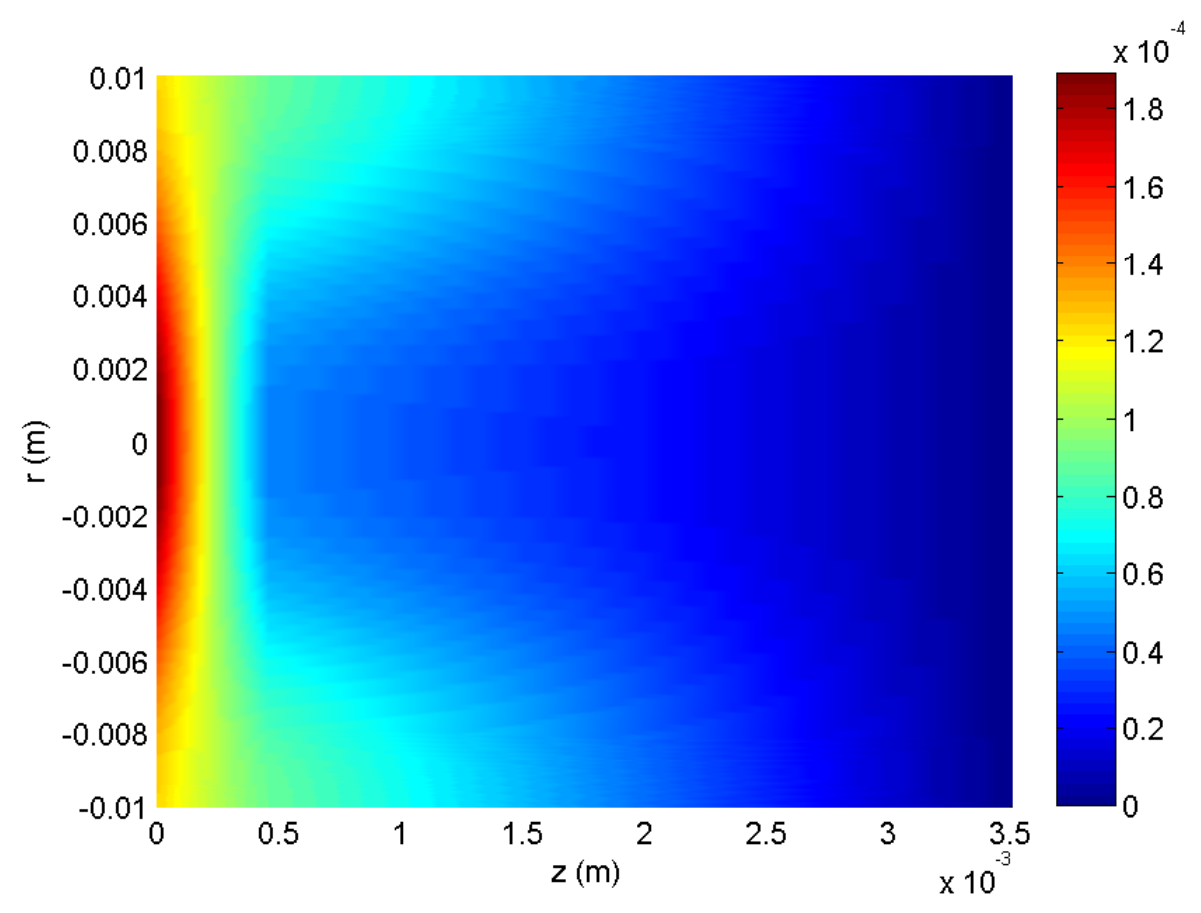

Figure 4. Simulation of the influence of the deposition of two gold layers on the electrical potential amplitude (V) distribution in a $\mathrm{Bi}_{2} \mathrm{Te}_{2.4} \mathrm{Se}_{0.6}$ sample subjected to a modulated Gaussian thermal excitation.

tion of all TE materials (with high or low electrical conductivity). In contrast to the $\mathrm{PTE}_{1 D}$ model, this new theory takes into account the contacts position as well as the laser beam radius. 


\section{B. Development of a photothermoelectric model taking into account the} Gaussian shape of the laser beam

Considering a TE sample subjected to a modulated heating on its front face $(z=0)$, see Fig.1. When the heating flux density displays a Gaussian distribution $(F(r, \omega)=$ $\left.\frac{F_{0}}{2} \exp \left(-r^{2} / R_{l}^{2}\right)\right)$, the temperature distribution in the sample as function of its depth $(z)$ and the exciting frequency can be written as:

$$
\bar{T}_{1(3 D)}(r, z, \omega)=2 R_{l}^{2} \int_{0}^{\infty} \bar{T}_{1(1 D)}\left(z, s_{1}(\lambda, \omega)\right) \exp \left(-\lambda^{2} R_{l}^{2} / 4\right) J_{0}(\lambda r) \lambda d \lambda
$$

This expression is obtained by applying the Hankel transforms and the Hankel inverse transforms to the equation describing the temperature distribution in the same sample when the thermal excitation is considered homogeneous ${ }^{7,8}\left(\bar{T}_{1(1 D)}\right)$. $F_{0}$ represents the heat flux density generated by the light source at the center of the light spot $(r=0$ and $z=0)$. $J_{0}$ is the zero-order Bessel function of the first kind, $\lambda$ represents Hankel's variable which is a superposition of planar waves with different spatial frequencies ${ }^{9} . s_{1}$ is a complex thermal wave number given by:

$$
s_{1}^{2}=\lambda^{2}+\sigma_{1}^{2}, \sigma_{1}=\frac{1+i}{\mu_{1}}, \mu_{1}=\sqrt{\frac{\pi f}{\alpha_{1}}}
$$

Here, $\alpha_{1}$ is the sample's thermal diffusivity. As the complex thermal wave number is dependent on $\lambda$, the thermal wave reflection coefficients in the case of a three dimensional (3D) heat propagation $\Gamma_{i j}$ are given by ${ }^{7,9}$ :

$$
\Gamma_{i j}(\lambda)=\frac{B_{i j}(\lambda)-1}{B_{i j}(\lambda)+1}, B_{i j}(\lambda)=\frac{\kappa_{i} s_{i}(\lambda)}{\kappa_{j} s_{j}(\lambda)}
$$

The indexes $i$ and $j$ refer to the different layers represented on figure 1. Indexes $0,1,2$ refer to the front layer, TE sample and the substrate, respectively. $\kappa$ is the thermal conductivity.

After replacing $\bar{T}_{1(1 D)}$ by its expression developed in reference ${ }^{2}$, the temperature distribution in the sample is given by:

$$
\begin{array}{r}
\bar{T}_{1(3 D)}(r, z, \omega)=2 R_{l}{ }^{2} \int_{0}^{\infty} \frac{F_{0}}{2 \kappa_{1} s_{1}\left(1+B_{01}\right)}\left[\frac{1+\Gamma_{12} \exp \left(-2 s_{1}\left(L_{1}-z\right)\right)}{1-\Gamma_{10} \Gamma_{12} \exp \left(-2 s_{1} L_{1}\right)} \exp \left(-s_{1} z\right)\right] \times \\
\exp \left(-\lambda^{2} R_{l}^{2} / 4\right) J_{0}(\lambda r) \lambda d \lambda
\end{array}
$$


Since the sample is a TE material that exhibits a Seebeck coefficient $\left(S_{1}\right)$, the generated voltage $(\Delta \bar{V})$ between two points located on its two faces $\left(z=0\right.$ and $\left.z=L_{1}\right)$ is given by:

$$
\begin{aligned}
\Delta \bar{V}(\omega)=\bar{V}_{1}\left(r=r_{0}, z=0, \omega\right)-\bar{V}_{1}\left(r=r_{L}, z=L_{1}, \omega\right) & =-S_{1} \times \\
& \quad\left[\bar{T}_{1}\left(r=r_{0}, z=0, \omega\right)-\bar{T}_{1}\left(r=r_{L}, z=L_{1}, \omega\right)\right]
\end{aligned}
$$

$r_{0}$ and $r_{L}$ are respectively the points at the surfaces $z=0$ and $z=L_{1}$ where the electrical potentials are measured.

By introducing eq.5 in eq.6, the photothermoelectric signal that the TE sample generates is expressed by:

$$
\begin{gathered}
\Delta \bar{V}_{(3 D)}(\omega)=2 R_{l}^{2} \int_{0}^{\infty} \frac{-S_{1} F_{0}}{2 \kappa_{1} s_{1}\left(1+B_{01}\right)}\left[\frac{1+\Gamma_{12} \exp \left(-2 s_{1} L_{1}\right)}{1-\Gamma_{10} \Gamma_{12} \exp \left(-2 s_{1} L_{1}\right)}\right] \exp \left(-\lambda^{2} R_{l}^{2} / 4\right) J_{0}\left(\lambda r_{0}\right) \lambda d \lambda \\
-2 R_{l}^{2} \int_{0}^{\infty} \frac{-S_{1} F_{0}}{2 \kappa_{1} s_{1}\left(1+B_{01}\right)}\left[\frac{\left(1+\Gamma_{12}\right) \exp \left(-s_{1} L_{1}\right)}{1-\Gamma_{10} \Gamma_{12} \exp \left(-2 s_{1} L_{1}\right)}\right] \exp \left(-\lambda^{2} R_{l}^{2} / 4\right) J_{0}\left(\lambda r_{L}\right) \lambda d \lambda \quad(7)
\end{gathered}
$$

This signal is dependent on the thermal properties of the TE sample, the electrical contacts positions and the laser beam radius. To remove any instrumental contribution which can affect this photothermoelectric signal, a normalization procedure should be performed. To achieve that, the substrate on which the TE sample is placed should be replaced by another substrate (e.g. air) having a known thermal effusivity. Hereinafter, the new signal generated by the TE sample when placed on air is written:

$$
\begin{gathered}
\Delta \bar{V}_{s(3 D)}(\omega)=2 R_{l}^{2} \int_{0}^{\infty} \frac{-S_{1} F(r, z=0, \omega)}{2 \kappa_{1} s_{1}\left(1+B_{01}\right)}\left[\frac{1+\Gamma_{10} \exp \left(-2 s_{1} L_{1}\right)}{1-\Gamma_{10}^{2} \exp \left(-2 s_{1} L_{1}\right)}\right] \exp \left(-\lambda^{2} R_{l}^{2} / 4\right) J_{0}\left(\lambda r_{0}\right) \lambda d \lambda \\
-2 R_{l}{ }^{2} \int_{0}^{\infty} \frac{-S_{1} F(r, z=0, \omega)}{2 \kappa_{1} s_{1}\left(1+B_{01}\right)}\left[\frac{\left(1+\Gamma_{10}\right) \exp \left(-s_{1} L_{1}\right)}{1-\Gamma_{10}^{2} \exp \left(-2 s_{1} L_{1}\right)}\right] \exp \left(-\lambda^{2} R_{l}^{2} / 4\right) J_{0}\left(\lambda r_{L}\right) \lambda d \lambda
\end{gathered}
$$

The ratio of $\bar{V}_{(3 D)}(\omega)$ to $\bar{V}_{s(3 D)}(\omega)$ gives the normalized photothermoelectric signal $\left(\bar{V}_{n(3 D)}(\omega)\right)$ free from all instrumental contributions:

$$
\bar{V}_{n(3 D)}(\omega)=\frac{\Delta \bar{V}_{(3 D)}(\omega)}{\Delta \bar{V}_{s(3 D)}(\omega)}
$$


When thermal effusivities of the front and back layers are known, the thermal diffusivity and effusivity of the TE material can be obtained by fitting eq.9 to the normalized experimental signal. This procedure will be referred in the following sections as $\mathrm{PTE}_{3 D}$ technique.

\section{EXPERIMENT}

To demonstrate the consistency of our theoretical developments, two TE materials with high and low electrical conductivities are elaborated and then thermally characterized using the PTE technique. In this part, we describe the samples preparation procedure and the experimental device of the PTE technique.

\section{A. Material synthesis}

As high electrical conductive material, a Bismuth selenido-telluride $\left(\mathrm{Bi}_{2} \mathrm{Te}_{2.4} \mathrm{Se}_{0.6}\right)$ sample was elaborated from pure elemental precursors ${ }^{10}$ (Alfa Aesar) of bismuth (needles, 99.99\%), tellurium (shots, 99.99\%), and selenium (shots, 99.99\%). Appropriate stoichiometric mixture of the elements were loaded in a $20 \mathrm{ml}$ tungsten carbide ball mill jar, containing seven tungsten carbide 10-mm-diameter balls. The mixture was then subjected to mechanical alloying for $30 \mathrm{~min}$ divided in 15 cycles of $2 \mathrm{~min}$ each at $700 \mathrm{rpm}$ in a Fritsch Pulverisette 7 (Fritsch $\mathrm{GmbH}$ ) premium line device. The obtained powder was compacted using a spark plasma sintering (SPS) process in a graphite die of $15 \mathrm{~mm}$ diameter at a pressure of 25 MPa during $30 \mathrm{~min}$ at a temperature of $723 \mathrm{~K}$.

As low electrical conductive material, a $\mathrm{Bi}_{2} \mathrm{Ca}_{2} \mathrm{Co}_{1.7} \mathrm{O}_{x}$ sample was prepared by the classical solid state method ${ }^{11}$. $\mathrm{Bi}_{2} \mathrm{O}_{3}$ (99.9\%, Aldrich), $\mathrm{CaCO}_{3}$ ( $\geq 99 \%$, Aldrich), and $\mathrm{Co}_{3} \mathrm{O}_{4}$ (99.5\%, Panreac) were mixed and milled in an agate ball mill for $2 \mathrm{~h}$ at $300 \mathrm{rpm}$ in acetone media. The resulting slurry was then sieved and the balls were washed with acetone to separate the grinding media and the powders mixture. The suspension was then totally dried under infrared radiation until a soft brownish powder was obtained. The dry mixture was then calcined twice at 750 and $800^{\circ} \mathrm{C}$ for $12 \mathrm{~h}$, with an intermediate manual milling, to decompose the $\mathrm{CaCO}_{3}$, uniaxially pressed at about $400 \mathrm{MPa}$ and sintered at $800^{\circ} \mathrm{C}$ for $24 \mathrm{~h}$ with a final furnace cooling. 


\section{B. PTE technique setup}

For each of the two studied materials, adapted samples were cut from the prepared rods. The $\mathrm{Bi}_{2} \mathrm{Ca}_{2} \mathrm{Co}_{1.7} \mathrm{O}_{x}$ sample was square-shaped with parallel opposite faces, its side and thickness are around $3 \mathrm{~mm}$ and $609 \mu \mathrm{m}$, respectively. The geometry of the $\mathrm{Bi}_{2} \mathrm{Te}_{2.4} \mathrm{Se}_{0.6}$ sample was round-shaped with parallel opposite faces, its diameter and thickness are about $15 \mathrm{~mm}$ and $450 \mu \mathrm{m}$, respectively.

In order to get equipotential surfaces on the $\mathrm{Bi}_{2} \mathrm{Ca}_{2} \mathrm{Co}_{1.7} \mathrm{O}_{x}$ sample, a highly electrically conductive coating (gold) was deposited by sputtering (Q150R rotary pumped coating system) on its two faces. This will avoid a 3D effect at this location. In the purpose of reducing as much as possible the 3D effect, thin gold layers were also deposited on the faces of the $\mathrm{Bi}_{2} \mathrm{Te}_{2.4} \mathrm{Se}_{0.6}$ sample. Then, two electrical leads connected to the electrodes were silver pasted and plugged into a EG\&G 7260 lock-in amplifier. Afterwards, the surfaces of the two studied samples were blackened in order to increase their light absorption.

To create a temperature difference and a voltage between the two faces of the TE sam-

ple, an internally modulated laser (Ventus VIS 532 nm, Laser Quantum) was employed to illuminate the front sample's face. For the frequency range used here $(1<f<100 \mathrm{~Hz})$, the temperature difference generated between the two sides of the sample is less than few hundreds of milli-Kelvin.

\section{RESULTS}

\section{A. Experimental results and discussion}

Amplitude and phase of the signal provided by the $\mathrm{Bi}_{2} \mathrm{Te}_{2.4} \mathrm{Se}_{0.6}$ sample are presented in Fig.5. The signal amplitude decreases with the exciting frequency because of the tempera-ture difference decreases between the two sides of the sample. Amplitude ratios and phase differences were calculated, providing the normalized amplitude and phase represented in Fig.6. As depicted on this figure, the model considering a 1D heat propagation in the sample $\left(\mathrm{PTE}_{1 D}\right.$ model) is not able to fit the normalized experimental signal. Conversely, the model taking into account the Gaussian shape of the excitation ( $\mathrm{PTE}_{3 D}$ model) described by eq.9 is successfully fitted to this experimental normalized signal using a non linear least square fitting procedure. To meet the requirements of the model (thermally thick substrates 

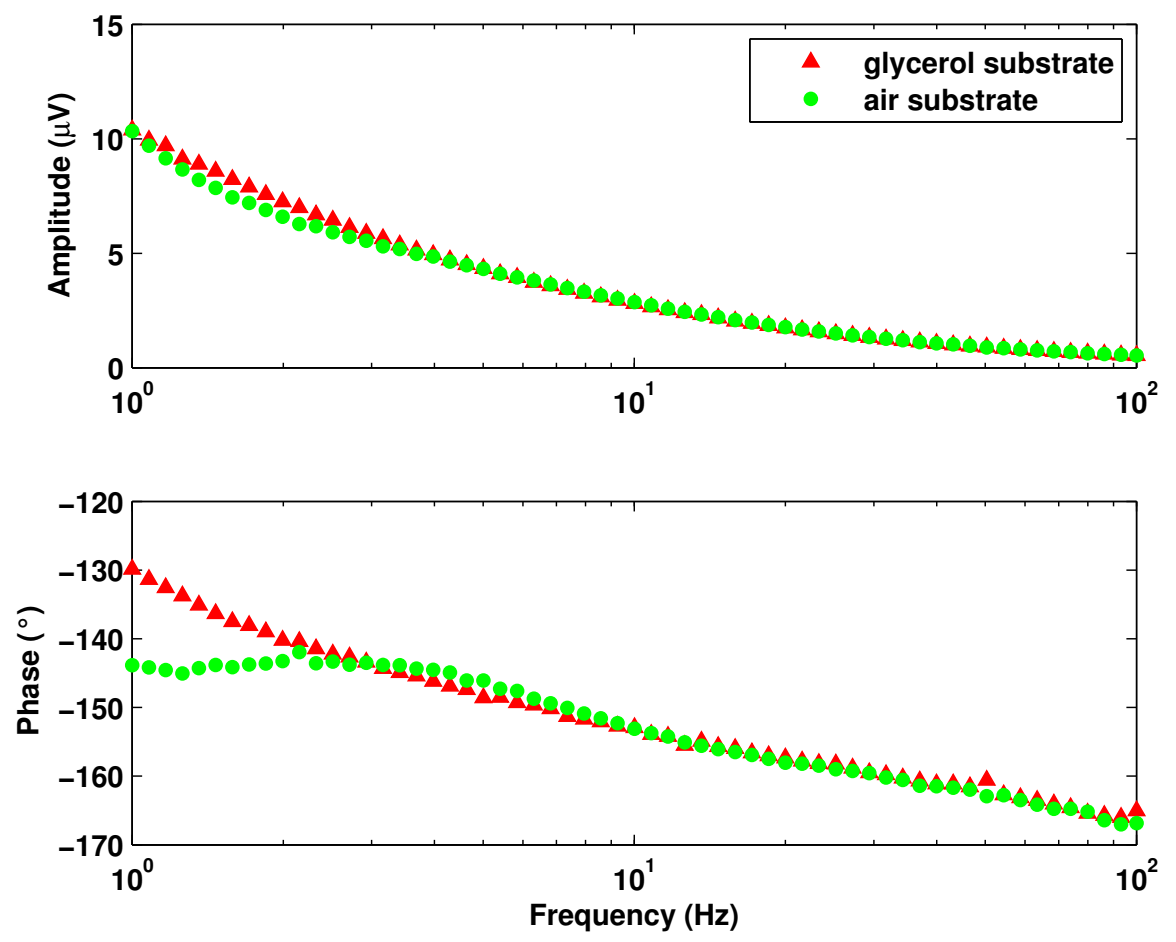

Figure 5. Frequency dependence of the photothermoelectric experimental signal (amplitude and phase) generated by the thermoelectric sample $\mathrm{Bi}_{2} \mathrm{Te}_{2.4} \mathrm{Se}_{0.6}$ at ambient temperature.

and negligible convection effect) and because of sensitivity, scans were performed in the frequency range of 1 to $100 \mathrm{~Hz}$.

From the numerical analysis of the experimental signal, a thermal diffusivity $\alpha_{1}=(8.71$ $\pm 0.3) \times 10^{-7} \mathrm{~m}^{2} \mathrm{~s}^{-1}$ and a thermal effusivity $e_{1}=(1582 \pm 72) \mathrm{W} \mathrm{s}^{1 / 2} \mathrm{~m}^{-2} \mathrm{~K}^{-1}$ were obtained. Calculus gives a thermal conductivity $k_{1}=(1.47 \pm 0.1) \mathrm{W} \mathrm{m}^{-1} \mathrm{~K}^{-1}$. The error values indicate the statistical uncertainties of the experimental results arising from the fit. The most-squares as well as the least-squares error analysis ${ }^{13}$ represented in Fig. 7 exhibit a single and identical minimum in the case of thermal diffusivity (Fig.7.a) and effusivity (Fig.7.b). This single minimum means that the calculated solution is unique (if the most squares error analysis shows a flat line, it indicates a multi-fold degeneracy which means that the fit parameter satisfies more than one solution for the same experimental data).

In order to validate this new technique, the obtained thermal parameters were compared to those obtained by the photothermal radiometry (PTR) on the same sample. The PTR technique is well described in the article of Depriester et $a .^{14}$. As it can be seen in table II, 

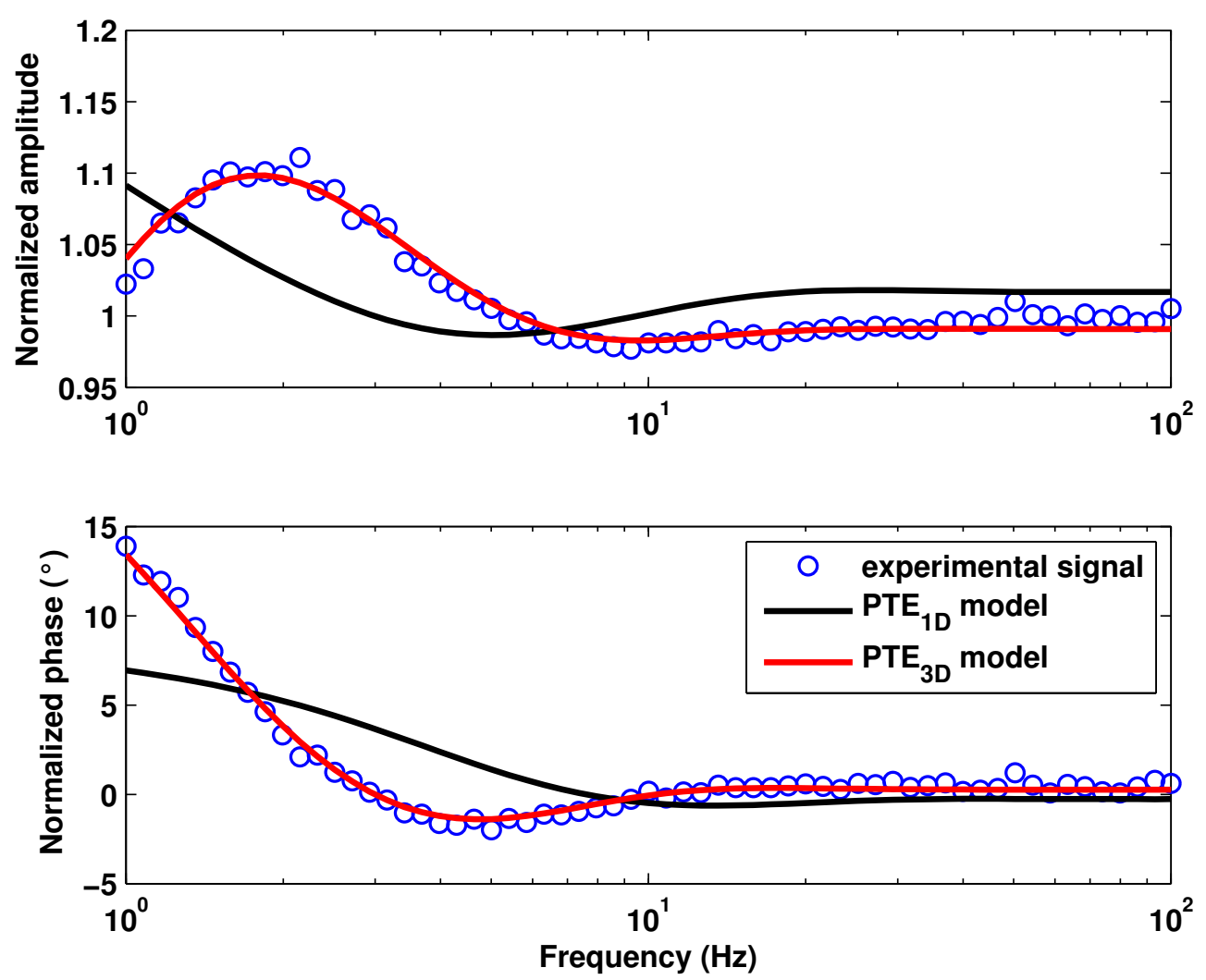

Figure 6. Normalized amplitude (upper plot) and phase (lower plot) of the photothermoelectric signal, plotted as a function of frequency. The circles represent the experimental data and the full lines represent the best fits of the $1 \mathrm{D}$ and the $3 \mathrm{D}$ models. The $\mathrm{Bi}_{2} \mathrm{Te}_{2.4} \mathrm{Se}_{0.6}$ sample has a thickness of $450 \mu \mathrm{m}$.

the $\mathrm{PTE}_{3 D}$ calculated thermal conductivity is in close agreement with the one obtained by the PTR technique. The deviation between the two conductivities is less than $4 \%$, confirming a good accuracy of the $\mathrm{PTE}_{3 D}$ technique. 

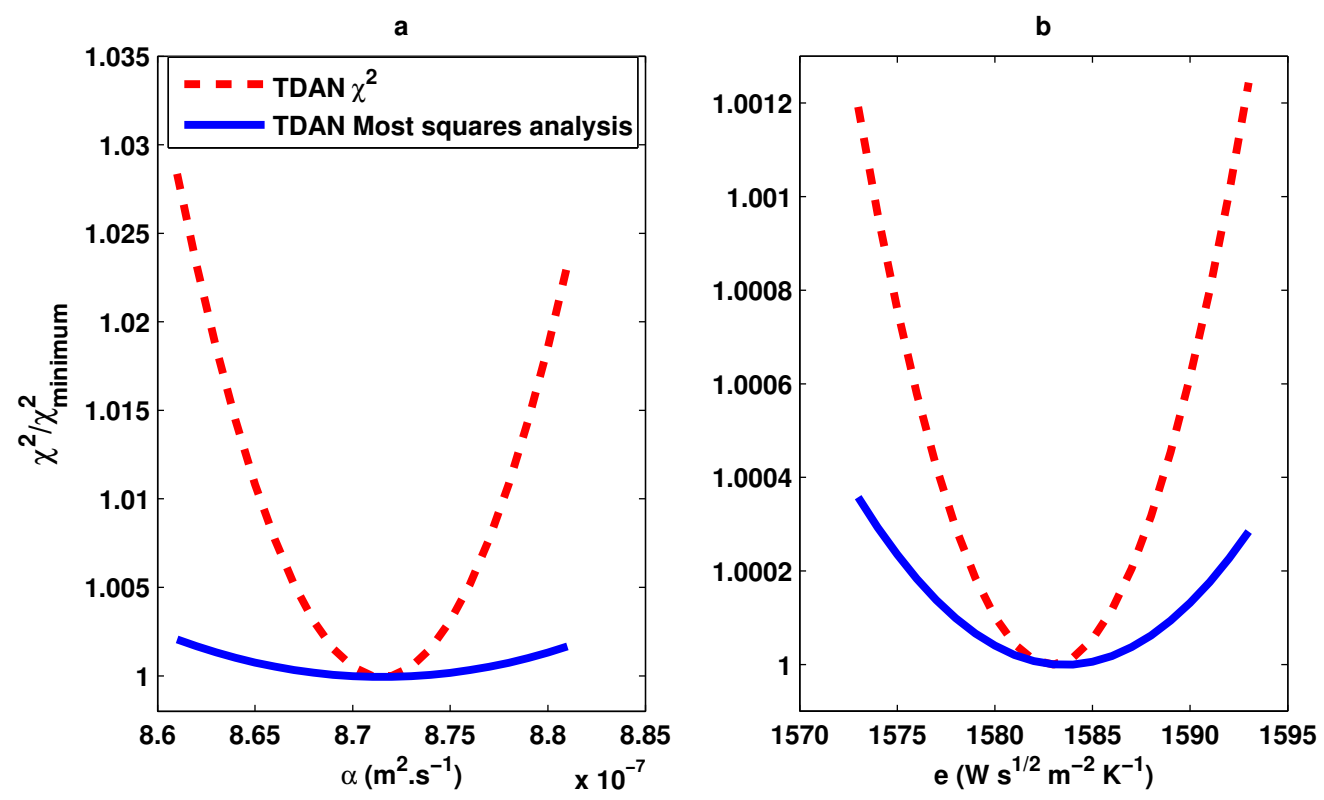

Figure 7. Most squares (full line) and least squares error analysis (dashed line) on thermal diffusivity (a) and effusivity (b) of the $\mathrm{Bi}_{2} \mathrm{Te}_{2.4} \mathrm{Se}_{0.6}$ sample.

\begin{tabular}{ccc}
\hline \hline & \\
Thermal parameters & $\mathrm{PTE}_{3 D}$ & PTR \\
\hline & & \\
$\alpha\left(10^{-7} \mathrm{~m}^{2} . s^{-1}\right)$ & $8.71 \pm 0.30$ & $9.79 \pm 0.02$ \\
\hline$e\left(\mathrm{~W} . \mathrm{s}^{1 / 2} \cdot \mathrm{m}^{-2} \cdot \mathrm{K}^{-1}\right)$ & $1582 \pm 72$ & $1432 \pm 08$ \\
\hline$\kappa\left(\mathrm{W} \cdot \mathrm{m}^{-1} \cdot \mathrm{K}^{-1}\right)$ & $1.47 \pm 0.10$ & $1.42 \pm 0.01$ \\
\hline \hline
\end{tabular}

Table II. Thermal parameters retrieved from $\left(\mathrm{PTE}_{3 D}\right)$ technique compared to those obtained by the PTR technique for a $\mathrm{Bi}_{2} \mathrm{Te}_{2.4} \mathrm{Se}_{0.6}$ sample at ambient temperature.

The experimental signals generated by the low conductive TE material $\left(\mathrm{Bi}_{2} \mathrm{Ca}_{2} \mathrm{Co}_{1.7} \mathrm{O}_{x}\right)$ are processed by following the same procedure already met in the case of the high conductive material, see Fig.8. As we deposited thin gold layers on the two faces of the sample, the $\mathrm{PTE}_{1 D}$ model was also fitted to the same experimental signal. The two models, fitting very well the normalized experimental signal, are quasi-superposed. The results obtained by using $\mathrm{PTE}_{3 D}$ and $\mathrm{PTE}_{1 D}$ are reported in table III. These results show that both PTE models $\left(\mathrm{PTE}_{1 D}\right.$ and $\left.\mathrm{PTE}_{3 D}\right)$ are suitable in the case of low conductive materials. In this 
case also, the PTR technique confirms the PTE results.
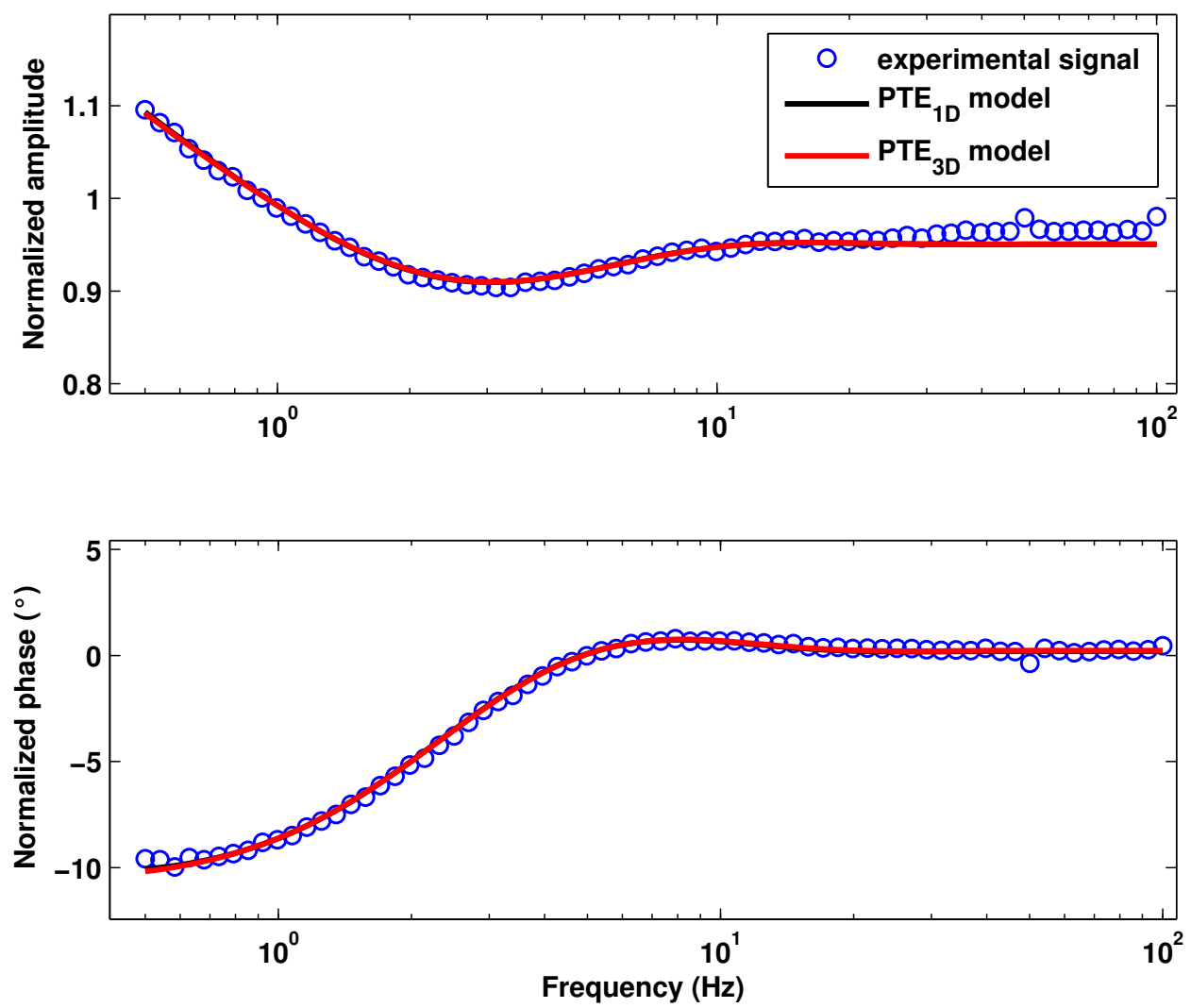

Figure 8. Normalized amplitude (upper plot) and phase (lower plot) of the photothermoelectric signal, plotted as a function of frequency. The circles represent the experimental data and the full lines represent the best fits of the $1 \mathrm{D}$ and the $3 \mathrm{D}$ models. The $\mathrm{Bi}_{2} \mathrm{Ca}_{2} \mathrm{Co}_{1.7} \mathrm{O}_{x}$ sample has a thickness of $609 \mu \mathrm{m}$. 


\begin{tabular}{cccc}
\hline \hline & & & \\
Thermal parameters & $\mathrm{PTE}_{1 D}$ & $\mathrm{PTE}_{3 D}$ & PTR \\
\hline & & & \\
$\alpha\left(10^{-7} \mathrm{~m}^{2} . s^{-1}\right)$ & $6.57 \pm 0.04$ & $6.67 \pm 0.15$ & $6.32 \pm 0.05$ \\
\hline$e\left(\mathrm{~W} . \mathrm{s}^{1 / 2} \cdot \mathrm{m}^{-2} \cdot \mathrm{K}^{-1}\right)$ & $1936 \pm 12$ & $1987 \pm 87$ & $2018 \pm 23$ \\
\hline$\kappa\left(\mathrm{W} . \mathrm{m}^{-1} \cdot \mathrm{K}^{-1}\right)$ & $1.57 \pm 0.02$ & $1.62 \pm 0.10$ & $1.60 \pm 0.02$ \\
\hline \hline
\end{tabular}

Table III. Thermal parameters retrieved from $\mathrm{PTE}_{3 D}$ and $\mathrm{PTE}_{1 D}$ techniques compared to those obtained by the PTR technique for a $\mathrm{Bi}_{2} \mathrm{Ca}_{2} \mathrm{Co}_{1.7} \mathrm{O}_{x}$ sample at ambient temperature.

\section{B. Conclusion}

In this work, we have reported a simple tool allowing an accurate thermal characterization of high efficient thermoelectric materials. After being restricted to low conductive TE materials, the PTE technique was extended to the most conductive materials.

Simulations using the finite element method have shown that it is possible to use the $\mathrm{PTE}_{1 D}$ model for the thermal characterization of materials with low electrical conductivities. This is possible by depositing thin conductive layers allowing the homogenization of the electrical potential distribution at the surfaces of the sample. When the TE materials electrical conductivity comes closer to the one of the deposited gold layer, which is the case for the most efficient TE materials, the deposit of a coating may be insufficient and the $\mathrm{PTE}_{1 D}$ model is then no longer valid.

In this work, we demonstrated that thermal parameters can then be extracted from the photothermoelectric signal by taking into account the Gaussian form of the thermal excitation source $\left(\mathrm{PTE}_{3 D}\right)$. The formalism of this new methodology is developed and a procedure for extracting thermal parameters is also proposed. Thermal parameters retrieved by the PTE technique are confirmed by those obtained by the conventional PTR technique. One of the main advantages of this approach is the non use of a temperature external sensor. In this work, it was also demonstrated that thermal parameters (thermal diffusivity, effusivity and conductivity) of these materials can be simultaneously determined.

This study paves the way for further developments. Once thermal parameters of a TE material are known, the relative evolution of Seebeck coefficient can be obtained, in princi- 
ple, from signal amplitude using the same configuration. Furthermore, transversal thermal conductivity could be obtained by the use of the $\mathrm{PTE}_{3 D}$ model. This will be the subject of our next investigations.

\section{ACKNOWLEDGMENTS}

This work was financially supported by "la région Hauts de France", "le Syndicat Mixte de la Côte d'Opale" and by the "Fond Européen de Développement Economique Régional". M. A. Madre and A. Sotelo acknowledge MINECO-FEDER (project MAT2013-46505-C3-1-R) and Gobierno de Aragon-FEDER (Grupo de Investigacion Consolidado T12) for financial support.

\section{REFERENCES}

${ }^{1}$ D. Rowe, Thermoelectrics Handbook : Macro to Nano 1014. isbn : 9780849322648 (CRC Press, 2006)

${ }^{2}$ M. Kuriakose, M. Depriester, R. Chan Yu King, F. Roussel, A. Hadj Sahraoui, J. Appl. Phys. 113, 044502 (2013).

${ }^{3}$ K. Touati, M. Depriester, M. Kuriakose and A. Hadj Sahraoui, New methodology for the thermal characterization of thermoelectric liquids. Review of Scientific Instruments 86 (2015)

${ }^{4}$ K.Touati, M.Depriester, A. Hadj Sahraoui, C. Tripon and D. Dadarlat, Combined photopyroelectric-photothermoelectric detection for thermal characterization of liquid thermoelectrics. Thermochimica Acta 642, 39-44. issn : 0040-6031 (2016)

${ }^{5}$ Goupil, C., Seifert, W., Zabrocki, K., Mller, E. and Snyder, G. J. Thermodynamics of Thermoelectric Phenomena and Applications. Entropy 13, 1481. issn : 1099-4300 (2011)

${ }^{6}$ Antonova, E. E. and Looman, D. C. Finite elements for thermoelectric device analysis in ANSYS in ICT 2005. 24th International Conference on Thermoelectrics, 2005. (juin 2005), $215-218$

${ }^{7}$ A. Mandelis, Diffusion-Wave Fields, Mathematical Methods and Green Functions, Springer Verlag New York, 2001.

${ }^{8} \mathrm{Qu}, \mathrm{H}$., Wang, C., Guo, X. and Mandelis, A. Reconstruction of depth profiles of thermal 
conductivity of case hardened steels using a three-dimensional photothermal technique. Journal of Applied Physics 104.

${ }^{9}$ Fabbri, L. and Cernuschi, F. Finite laser beam size effects in thermal wave interferometry. Journal of Applied Physics 82, 5305-5311 (1997)

${ }^{10}$ Lognoné, Q. et al. Quantitative Texture Analysis of Spark Plasma Textured n-Bi2Te3. Journal of the American Ceramic Society 97, 2038-2045. issn : 1551-2916 (2014)

${ }^{11}$ M.A. Madre, Sh. Rasekh, K. Touati, C. Salvador, M. Depriester, M.A. Torres, P. Bosque, J.C. Diez, A. Sotelo, From nanosized precursors to high performance ceramics: The case of Bi2Ca2Co1.7Ox, Materials Letters, Volume 191, 15 March 2017, Pages 14-16, ISSN 0167-577X, http://dx.doi.org/10.1016/j.matlet.2017.01.031.

${ }^{12}$ Sotelo, A. et al. Solution-based synthesis routes to thermoelectric Bi2Ca2Co1.7Ox. Journal of the European Ceramic Society 31, 1763-1769. issn : 0955-2219 (2011)

${ }^{13}$ Menon, P. C., Rajesh, R. N. and Glorieux, C. High accuracy, self-calibrating photopyroelectric device for the absolute determination of thermal conductivity and thermal effusivity of liquids. Review of Scientific Instruments 80 (2009)

${ }^{14}$ Depriester, M., Hus, P., Delenclos, S. and Sahraoui, A. H. New methodology for thermal parameter measurements in solids using photothermal radiometry. Review of Scientific Instruments 76 (2005) 